\title{
Estudio sobre la experiencia de hombres atendidos en centros residenciales de ayuda mutua para la atención de las adicciones
}

\author{
Rodrigo Marín-Navarrete, ${ }^{1,2}$ Angélica Eliosa-Hernández, ${ }^{2}$ Ignacio Lozano-Verduzco, ${ }^{2}$ \\ Carla Fernández-De la Fuente, ${ }^{2}$ Bernardo Turnbull, ' Antonio Tena-Suck'
}

Artículo original

\section{SUMMARY}

Addictive disorders and behaviors have increased in the past years in Mexico. These behaviors and disorders are considered a public health issue because of the social and economic strains they generate. However, the state is unprepared to attend such high demands. Non government organizations have arisen in order to fulfill this demand, but it is known that many of them do not regard federal health regulations and often they have infringed the basic human rights. The present study has for objective to analyze the experience of men who have been atended in self-help residential substance abuse attention centers. Fifteen focalized interviews were carried out with men of different characteristics, all of them having been attended in at least two centers. The results help to understand the consumption dynamic, usually linked to family abandonment and anger. It further analyzes the unhealthy and inhuman services offered in many of these centers such as poor feeding and hygiene services; many of the men are admitted violently and against their will. It is concluded that even though these centers respond to the demand that addictive disorders have, they do not professionally and skillfully support a rehabilitation process. It is thus of utter importance to guarantee the training and proffessionalization of these centers' staff through an observation and regulation process.

Key words: Addictions, treatment, self-help, men.

\section{RESUMEN}

Los trastornos y conductas adictivas se han incrementado en México en los últimos años. Estas conductas y trastornos son considerados un problema de salud pública debido a los estragos sociales y económicos que implican. Sin embargo, la demanda de atención ha rebasado las capacidades del Estado, por lo que han surgido organizaciones civiles que buscan responder a dicha problemática, aunque en muchas ocasiones esto suele hacerse sin atender a la reglamentación federal para la atención de la salud y las adicciones, por lo que existen casos en los que se han violentado los derechos humanos básicos. El presente estudio tuvo por objetivo analizar la experiencia de hombres atendidos en centros residenciales de ayuda mutua para la atención de las adicciones. Se llevaron a cabo 15 entrevistas focalizadas con hombres de diferentes características, que habían sido internados en al menos dos de estos centros. Los resultados ayudan a comprender la dinámica de consumo, que usualmente está ligada al abandono familiar y al enojo. Además, se analiza el uso de servicios de estos centros, como alimentación e higiene. Se encontró que muchos de estos hombres fueron ingresados de manera involuntaria y se vieron expuestos a diversas formas de abuso. Se concluye que a pesar de que estos centros responden a una necesidad de la sociedad para atender los problemas de salud que generan las adicciones, es imprescindible garantizar el respeto a los derechos humanos, asegurando la regulación y reglamentación de los centros.

Palabras clave: Adicciones, tratamiento, ayuda mutua, hombres.

\section{INTRODUCCIÓN}

El consumo de sustancias psicoactivas afecta de modo significativo la calidad de vida de las personas y, debido al elevado costo social y económico que éste produce, se le considera un problema de salud pública. En México, la Encuesta Nacional de Adicciones (ENA) señala que el número de personas que probaron una droga alguna vez en la vida ascendió de 3.5 millones en 2002 a 4.5 millones en 2008. Sin embargo, para 2011 no se reportaron incrementos significativos en el consumo de drogas, no siendo así para el consumo de alcohol, el cual presentó un incremento. Los jóvenes y los adultos jóvenes son los más afectados por el uso de drogas, pues se ha identificado que las generaciones actuales se encuentran más expuestas a ellas, ya que se ha reportado que el riesgo de experimentación se incrementa considerablemente cuando un amigo o familiar las utiliza (2.69 y 3.78 veces, respectivamente) ${ }^{1.4}$

El mismo estudio revela que cerca de $10 \%$ de los consumidores de sustancias acudió a tratamiento en el último año y

Departamento de Psicología de la Universidad Iberoamericana, Ciudad de México.

2 Unidad de Ensayos Clínicos, Instituto Nacional de Psiquiatría Ramón de la Fuente Muñiz.

Correspondencia: Rodrigo Marín-Navarrete. Unidad de Ensayos Clínicos, Sub-Dirección de Investigaciones Clínicas, Instituto Nacional de Psiquiatría Ramón de la Fuente Muñiz. Calz. México-Xochimilco 101, San Lorenzo Huipulco, Tlalpan, 14370, México DF. Teléfono: (55) $4160-5480$ y 5481. E-mail: rmarin@inprf.gob.mx

Recibido: 14 de diciembre de 2012. Aceptado: 27 de mayo de 2013. 
los recursos de atención más utilizados por esta pequeña parte de la población fueron: los grupos de ayuda mutua (53.8\%), la atención psicológica (45.1\%) y la psiquiátrica $(40.1 \%) .{ }^{3}$ De acuerdo con lo reportado por la Secretaría de Salud en 2008, se calcula que existen aproximadamente 500000 personas consumidoras de drogas ilegales que requieren atención especializada y alrededor de cuatro millones que requieren una intervención breve, y ello sin incluir las necesidades de tratamiento para tabaco y alcohol, de tal forma que el incremento de los trastornos por consumo de sustancias psicoactivas ha impactado significativamente en los últimos años, lo que ha elevado las necesidades de atención. ${ }^{1}$

En México, como en otros países, los problemas derivados del consumo de alcohol y drogas exceden muchas veces los recursos asignados por el Estado para atenderlos. ${ }^{5}$ Esta circunstancia ha generado diversas formas de organización por parte de la sociedad civil encaminadas a suplir las carencias del financiamiento público..$^{6-8}$

Una de las formas más usuales que ha tomado la organización social, en muchos países, para responder al problema de las adicciones son los grupos de ayuda mutua. Estos grupos reúnen individuos que comparten una misma problemática, se apoyan colectivamente $\mathrm{y}$, eventualmente, resuelven su problema. En su mayoría, emplean variantes del modelo de los doce pasos, desarrollado originalmente por Alcohólicos Anónimos el 10 de junio de 1935 en la ciudad de Akron, Ohio, E.U. ${ }^{9-11}$

En México existen registrados poco más de 14000 grupos de la "Central Mexicana de Servicios Generales de Alcohólicos Anónimos A.C.", 12 y otros 2402 grupos de la "Sección México de Alcohólicos Anónimos A.C.", 13 sin considerar otras variaciones como los grupos de "AA 24 horas de Terapia Intensiva", 14 "Grupos 24 horas de Alcohólicos Anónimos",15 "Grupo Jóvenes de Alcohólicos Anónimos"16 y "Narcóticos Anónimos Región México". ${ }^{17}$ Lo anterior sitúa a México como el tercer país con más grupos de AA después de Estados Unidos y Canadá. ${ }^{11}$

Asimismo, diversas sociedades médicas especializadas han reconocido la valiosa aportación de los Grupos de Ayuda Mutua a la rehabilitación de las personas que sufren algún tipo de dependencia. Éstas señalan que, cuando el paciente recibe tratamiento formal y se involucra en el programa de los 12 pasos, obtiene mejores resultados que cuando asiste únicamente a alguna de estas maniobras de atención. ${ }^{10}$ No obstante, los Grupos de Ayuda Mutua no son considerados un tratamiento formal ya que no son dirigidos por profesionales de la salud y sus procedimientos carecen de evidencia y validación científica. ${ }^{18,19}$ Sin embargo, es evidente que representan un importante complemento del tratamiento profesional, más que una sustitución del mismo. ${ }^{20}$

Aunado a lo anterior, los "Grupos Tradicionales", * es decir, los grupos de tipo ambulatorio cuyas sesiones tienen una duración de hora y media, no parecen ser efectivos en los casos de personas que presentan una alta gravedad de la adicción, así como trastornos mentales comórbidos. ${ }^{20}$ Esta situación de ineficacia no sólo se presenta en la atención de la Ayuda Mutua tradicional sino en general en los tratamientos ambulatorios, ya que la gravedad del problema requiere en muchos casos una contención residencial, misma que implica al menos desintoxicación, estabilización de la sintomatología psiquiátrica aguda y estabilización psicológica integral (cognición, emoción y conducta). Con este antecedente y debido a las reducidas ofertas profesionales del sector público y lo costosas e inaccesibles que son las ofertas del ámbito privado para la atención residencial de las adicciones, la sociedad civil se vio en la necesidad de realizar adaptaciones a los "Grupos Tradicionales", creando agrupaciones que ofrecen atención residencial basada en el modelo de la ayuda mutua cuyos servicios han sido dirigidos principalmente a las personas de escasos recursos. Dichas agrupaciones recibieron el nombre de "Anexos", los cuales dieron origen al modelo de los Centros Residenciales de Ayuda Mutua para la Atención de las Adicciones (CRAMAA), ${ }^{* *}$ mismos que han adoptado la filosofía de los 12 pasos desarrollados por Alcohólicos Anónimos (AA). Sin embargo, distan mucho de ser iguales a los "Grupos Tradicionales", ya que los CRAMAA refieren no adherirse a las 12 tradiciones de AA, pero sí a la filosofía de recuperación. ${ }^{11}$

En referencia a lo anterior, la Encuesta Nacional de Adicciones 2011 señala que la asistencia a los llamados CRAMAA disminuyó para la población masculina (36.4 a $30.5 \%)$. También es cierto que se ha observado un incremento en el uso de estos servicios para las mujeres (42.9 a 53.7\%), además de que continúan siendo una opción para una tercera parte de la población afectada $(32.1 \%) .^{3}$

Con lo anterior se hace evidente que los CRAMAA se han convertido en una opción de primera mano para familiares y pacientes que sufren de trastornos relacionados con el consumo de alcohol y otras drogas. La principal característica de estos centros es su heterogeneidad, ya que la mayoría ofrece una diversidad de servicios residenciales con una duración variable que puede ir de cuatro semanas hasta doce meses. Otra de las características relevantes es su infraestructura; algunos cuentan con instalaciones amplias, mientras que otros, debido al espacio limitado y la demanda de usuarios, sufren de hacinamiento. Además, es importante mencionar que la estructura jerárquica de estos centros se encuentra constituida por individuos que han logrado mantenerse sin consumo por mayor tiempo y buscan compartir su experiencia induciendo la recuperación en otros. Sin embargo, gran parte de dichos centros no cuentan con el apoyo de especialistas y/o profesionales de la salud. ${ }^{9}$

\footnotetext{
* "Grupos Tradicionales" es el término que los miembros de Alcohólicos Anónimos han adoptado para refrerirse a los grupos originales de AA.

* * "CRAMAA" es un acrónimo que se propone para referirse a los Centros Residenciales de Ayuda-Mutua para la Atención de las Adicciones.
} 
Si bien es cierto que los CRAMAA representan una alternativa valiosa para muchas personas que necesitan superar su problema de consumo de sustancias, también es relevante señalar que una de sus principales limitaciones es que, en su mayoría, violan la Ley General de Salud al operar sin equipo, personal e infraestructura adecuados según los lineamientos marcados por la Norma Oficial Mexicana NOM-028-SSA2-2009 para la prevención, tratamiento y control de las adicciones, además de que se carece de un censo real, actual y oficial de dichas organizaciones. ${ }^{21,22}$

Por otro lado, es común que el personal que labora en estas agrupaciones no cuente con un adiestramiento especializado que le permita llevar a cabo una evaluación sistemática de sus procedimientos y resultados. Esto trae como consecuencia que las personas que ingresan no sean diagnosticadas, por lo que se asume únicamente la presencia de un problema por consumo de sustancias, desconociéndose la alta correlación de trastornos mentales comórbidos tal y como lo reporta la evidencia científica. ${ }^{23}$ A su vez, esto tiene graves implicaciones en la calidad de vida del individuo, traduciéndose en un mayor índice de recaídas, ingresos y reingresos a unidades de atención, así como un incremento en las conductas de riesgo y deterioro biopsicosocial.

De acuerdo con cifras de la Secretaría de Salud, en el país existen cerca de 20000 camas para proporcionar tratamiento residencial, de las cuales se calcula que sólo 4000 cumplen con la aplicación de la NOM-028-SSA2-1999. Por otro lado, se han registrado alrededor de 1730 centros de atención o rehabilitación para pacientes en internamiento. De éstos, 10 corresponden a Centros de Integración Juvenil, 20 a centros estatales, 400 a clínicas privadas y se estima la existencia de al menos 15000 grupos que operan bajo el modelo de Ayuda Mutua de Alcohólicos Anónimos; sin embargo, carecen de un protocolo establecido de atención. Asimismo, en relación con los CRAMAA, en el Distrito Federal existen sólo 65 unidades de atención residencial que se apegan a los lineamientos de la NOM-028-SSA2-2009.22

Desde hace varios años numerosos estudios han puesto en evidencia la falta de claridad en relación con los procedimientos terapéuticos, así como el maltrato físico y emocional que tiene lugar al interior de los CRAMAA. ${ }^{9,24-26}$

Adicional a los estudios científicos, dicha situación ha sido ampliamente documentada en fuentes no oficiales como notas periodísticas en prensa escrita, radio y televisión, donde se describen las condiciones atentatorias de los derechos humanos prevalecientes en varios de estos lugares conocidos como "Anexos". Entre ellas destacan: hacinamiento, insalubridad, agresiones físicas y verbales, abuso sexual, tortura, privación de la libertad, explotación y esclavitud.

Es por lo anterior que el objetivo del presente estudio fue recuperar la experiencia de los usuarios de los servicios en los CRAMAA, así como la dinámica de consumo y proceso de internamiento, resaltando la forma de funcionamiento y uso de servicios al interior de los centros.

\section{MÉTODO \\ Participantes}

Se realizaron entrevistas semiestructuradas focalizadas ${ }^{27}$ a hombres que tenían la experiencia de haber ingresado en al menos dos CRAMAA, que opcionalmente estuvieran recibiendo atención en alguno de estos centros al momento de la entrevista y que accedieran de manera voluntaria a participar en el estudio. En promedio, los entrevistados tenían 40 años, con una desviación estándar de 12.18, en un rango de edad entre 23 y 58 años. Dos terceras partes contaban con estudios de licenciatura o posgrado y una tercera parte tenía estudios de preparatoria o secundaria. El 60\% estaba separado o divorciado, una quinta parte se encontraba en unión libre o en pareja y el resto era soltero.

\section{Análisis de datos}

La interpretación de los datos siguió el método fenomenológico-interpretativo mediante un análisis de contenido de significados, desde la óptica de la salud pública. Se realizó un libro de códigos que se desprenden de los objetivos de la investigación y que fueron enriquecidos con las narraciones de cada entrevista, logrando la "Saturación teórica" a los 15 participantes. Se utilizó el software ATLAS.ti versión 5.5 y NVIVO versión 9.0, para el análisis de los datos.

\section{RESULTADOS}

Del análisis de datos surgieron los siguientes grupos o ejes temáticos como los más significativos: a) Dinámica de consumo; b) Proceso de internamiento; c) Experiencia de los usuarios en los CRAMAA; d) Uso de Servicios.

\section{A) Dinámica de consumo}

En general, el consumo de alcohol, tabaco y otras drogas se presenta durante la adolescencia, ya que están involucrados diversos cambios en el funcionamiento psicosocial que pueden constituir factores de riesgo o protección que facilitan o retrasan el consumo de sustancias..$^{28,29}$ En México, se ha observado que el inicio de consumo de tabaco y alcohol se reporta en edades cada vez más tempranas, lo cual incrementa la probabilidad de usar otras drogas y progresar a la dependencia. ${ }^{1}$

$\mathrm{El}$ anterior supuesto teórico fue corroborado en las narraciones de los participantes del estudio, ya que refirieron haber comenzado el consumo de sustancias en edades muy tempranas, oscilando entre los siete y 15 años de edad.

\footnotetext{
"La primera vez que consumí fue a los... siete años...siete o nueve años no me acuerdo bien..." (Efraín, 39 años).

"Yo empecé con el consumo de alcohol a los doce años, a esa edad fue mi primera borrachera." (José Ramón, 35 años).
} 
"Yo empecé a consumir alcohol a los 15 años...iba en tercero de secundaria y fue con unos amigos..." (Jerónimo, 39 años).

Diversos factores se han asociado con el riesgo en el inicio del uso de drogas. Por ello, es de vital importancia considerar que tanto la exposición como la disponibilidad incrementan la probabilidad del consumo en los adolescentes. Se estima que cerca de la mitad de jóvenes que han estado expuestos a la oportunidad del uso de drogas las han consumido. ${ }^{1}$

Por otra parte, cabe señalar que la disponibilidad no se limita al acceso físico de las sustancias, ya que las normas sociales y el entorno inmediato tienen un fuerte impacto en el inicio y mantenimiento del consumo de sustancias. ${ }^{2}$ El análisis de los resultados sugiere que el consumo inicial de sustancias se ve influido en gran medida por la relación familiar, la cual en ocasiones incrementa el riesgo en los usuarios para la búsqueda de nuevas relaciones sociales permeadas, frecuentemente, por el consumo.

"...Un viernes saliendo de la secundaria (mis amigos) me invitaron a que fuéramos a tomar unos tequilas... ahí fue la primera vez que consumi, después de eso, seguí tomando con ellos en fiestas y reuniones." (Jerónimo, 39 años).

"...hice mi primer contacto con el alcohol, ha de haber sido brandy porque era lo que tomaba mi papá. Tomé una botella y me fui con mis hermanos, amigos y vecinos a una azotea de alguna de las casas y ahí estuvimos bebiendo hasta que nos emborrachamos ..." (Efrain, 39 años).

"Llegaron los meros meros que se juntaban con la banda, llegaron con solvente, pero a mí no me ofrecieron, sin embargo a mi me llamaba la atención cómo se activaban con la 'mona'. Los veía risa y risa, los veía contentos, por eso me llamó la atención." (Pablo, 39 años).

Otro hallazgo consistió en que los participantes reportaron un tipo de distanciamiento de la familia, así como un acercamiento a diversos grupos de riesgo, situaciones que impactan sobre el consumo, con lo que se incrementa tanto la frecuencia como la cantidad, aspectos que se traducen con el tiempo en el desarrollo de un trastorno por dependencia a sustancias.

\footnotetext{
"Al principio compraba lo que eran $\$ 5$ pesos de 'mota' o sea lo que ahorita serían como unos $\$ 20$, pero después de un rato ya no me satisfacía esa cantidad, se me hacía muy poquito." (Pablo, 39 años).

"De ahí empecé a tomar los días viernes; después viernes y sábado; después viernes, sábado, domingo y lunes; hasta llegar a tomar un mes completo durante todo el día... también recuerdo que estar con gente que consumía en grandes cantidades hacía que yo bebiera más; yo calculo que consumía hasta 5 litros en un día, más aparte otro litro en la noche para controlarme y para curármela..." (Jerónimo, 55 años).
}

Posteriormente, el usuario comienza el consumo en solitario, por tanto, a medida que se incrementa el consumo, también los problemas asociados a éste, llevándolo a un deterioro biopsicosocial que manifiesta la necesidad de atención.

"A causa de mi consumo me divorcié y me quedé en la calle; me quería morir, fui muy cobarde para suicidarme y me seguí drogando." (Ricardo, 53 años).

"Ya tenía mi propio negocio, entonces me llevaba la botella al negocio y ahí me la pasaba bebiendo y dizque trabajando, pero llegó el momento en que ya no podía trabajar, lo perdí todo y empecé a vivir de la familia, de los hermanos y amigos..." (Alfonso, 45 años).

Es por lo anterior que las personas significativas del consumidor acumulan diversas razones de internamiento que justifican ingresar al paciente en un centro residencial y en muchas ocasiones incluso en contra de su voluntad (figura 1).

"...después de varios días en la calle, finalmente no sé cómo mi mamá me encontró y me metió a un 'anexo'. Yo nunca, nunca había conocido un lugar de esos, ahora entiendo que ella tampoco sabía cómo ayudarme y nada más me llevó y me encerró..." (Joel, 24 años).

"mi mamá se cansó de pagar internamientos y yo recaía constantemente, ella ya se había acabado todo su dinero en mi recuperación, hasta que un compañero que también es de AA le dijo 'mire vecina, ya no pague, conozco un lugar en el cual la estancia es gratis, ahí nada más lleva de vez en cuando una despensita y a sus tres meses lo saca' y así fue..." (Gaby, 31 años)

"Al principio y casi siempre ha sido mi mamá la que me llevaba. Ella me decía '¿por qué no puedes controlarte? ¡Trata de controlarte!', y yo siempre tenía miles de pretextos, y en verdad que intentaba controlarme pero no podía y por eso ella me 'anexaba'." (Alfonso, 45 años).

\section{B) Proceso de internamiento}

La evidencia reporta que es poco común que el consumidor sea el primero en buscar ayuda profesional por sí mismo. Esto se debe a la evolución natural de la enfermedad, que implica una pobre o nula conciencia de enfermedad en el paciente. Es así que con frecuencia el primer ingreso a tratamiento es referido como una petición o imposición de la familia ya que, de acuerdo con las entrevistas realizadas, los miembros de la familia son los primeros en darse cuenta de que la situación de consumo se ha vuelto disfuncional. Para ello toman como indicadores el deterioro físico, el abandono escolar y/o laboral, el distanciamiento familiar, la conducta temeraria y antisocial, entre otros.

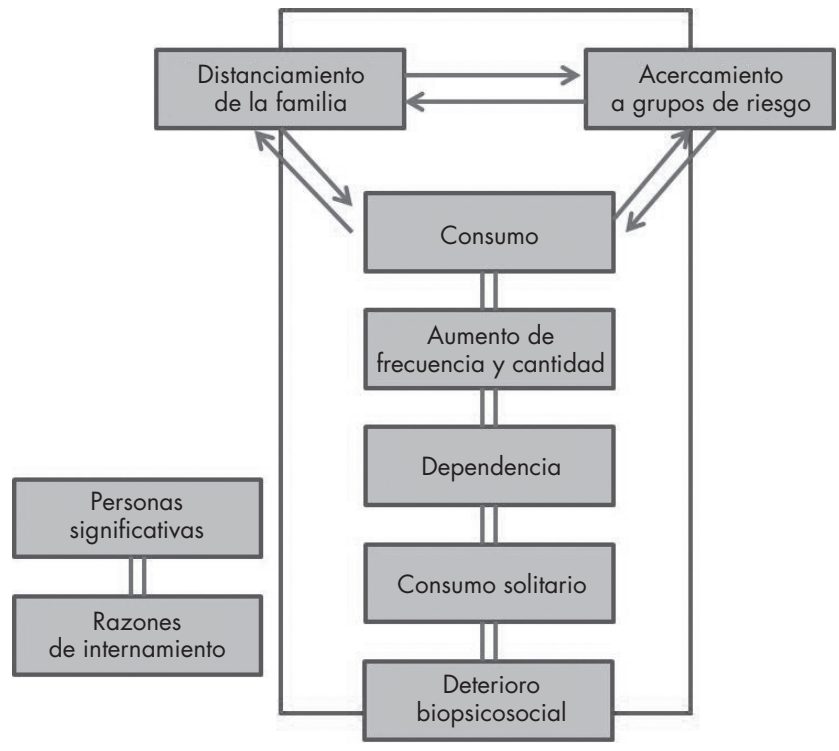

Figura 1. Dinámica de consumo. 
Sin embargo, estudios realizados en México sugieren que en promedio el primer contacto de atención, en el caso de los hombres, es de 8.8 años después del inicio en el consumo de alcohol o drogas, por lo que resulta evidente la gravedad en el deterioro biopsicosocial del individuo. ${ }^{30}$

Inicialmente, los usuarios reportan que la familia busca soluciones rápidas y entonces deposita la confianza en remedios caseros, yerbas, juramentos y promesas, entre otros. Posteriormente, los familiares, al no obtener respuestas efectivas y eficaces para resolver su problemática, recurren al apoyo de instituciones a las que acuden por recomendación de algún otro familiar, conocido y/o profesional no especializado en el área o incluso a través de las líneas públicas de orientación telefónica.

"Entonces cuando me da de alta el doctor...de manera muy directa, se me
queda viendo y me dice 'es usted alcohólico, y necesita ir a Alcohólicos
Anónimos' y le da dos tarjetas a mi mamá de dos grupos, uno donde inter-
nan y otro a donde se asiste a pláticas en la tarde o en la noche." (Alfonso,
28 años).
"... (mi mamá) habló a 'Locatel' y a 'Vive sin Drogas'... le dijeron: 'mire
hay una casa así como para lo que usted busca...no es muy caro, y si
quiere puede ir a ver las instalaciones'. Finalmente le dieron los teléfonos
y mi mamá marcó al centro y pidió informes para internarme..." (Gaby,
31 años).

Los usuarios mencionaron que durante los primeros internamientos no era tan evidente para ellos la problemática asociada al consumo, ya que aún mantenían cierta funcionalidad en determinadas áreas de sus vidas. Es por lo anterior que al momento en que se plantea la alternativa de internamiento ellos sienten que aún no es tiempo, que no están listos e incluso se perciben demasiado jóvenes para tener un problema serio relacionado con el consumo de sustancias, por lo que, con frecuencia, los familiares promueven el internamiento involuntario.

"Pues yo todavía no estaba listo, a pesar de que me drogaba, tenía un buen trabajo y un buen ingreso económico, yo no estaba dispuesto a dejar mi vida." (Ricardo, 53 años).

"Ya casi al final, en los últimos dos meses, fue cuando me empecé a dar cuenta que ya no tenía control; empecé a tomar cosas que no eran mías y a ver mi vida entorno a la sustancia, sin embargo no quería internarme." (Joel, 24 años).

Por otro lado, es importante señalar que dicha involuntariedad en el ingreso a los centros despierta en el usuario diversas emociones, entre ellas, enojo y coraje, lo cual ocasiona frecuentemente que el paciente escape del proceso de internamiento, situación que incluso reportan asociarlo a las reincidencias del consumo.

"Yo llegué a los internamientos cada vez con más resentimiento, cada vez más enojado... con menos ganas de recuperarme, o sea estaba en un internamiento porque me obligaban, pero no porque yo quisiera estar y muchas veces tenía el deseo y la fantasía de llevar a mis papás y hermanos a internarlos o llevar a mi pareja a internarla para que sintieran lo que yo estaba sintiendo." (Ricardo, 53 años).

Adicionalmente, los entrevistados reportaron que a pesar de estar conscientes de los problemas y la falta de control sobre su consumo, la sustancia cumplía una función, por lo que, además de evitar o aminorar los síntomas negativos del síndrome de abstinencia, permitía al usuario obtener algunas ganancias secundarias; entre ellas, diversión, placer, pertenencia a un grupo social y compañía.

"No aceptaba que... el consumo de drogas y alcohol me estaban haciendo daño, me estaban despojando de muchas cosas y a pesar de que los compañeros de grupo me decían que cada recaída sería peor y las consecuencias serían más severas; yo no hacía caso." (Gaby, 31 años).

Finalmente, se encontró que existen múltiples elementos que acompañan al internamiento involuntario y que además, de acuerdo con lo que reportaron los entrevistados, impacta negativamente en su percepción de la rehabilitación. Por ejemplo, el ser víctimas de abuso constante al interior del centro es referido como uno de los precipitadores de reincidencia al consumo una vez que éstos egresan del CRAMAA. Por otro lado, existe un constante descontento ya que el internamiento es vivido como la inclusión a un sistema de vigilancia, control y sometimiento (figura 2).

\section{C) Experiencia en los CRAMAA}

Durante el trabajo de campo, se hizo evidente la presencia de dos tipos principales de CRAMAA. El primer grupo fue definido como "light" caracterizado por la estancia voluntaria al interior del centro, mejor uso de servicios y la evitación de la violencia como medio de control. El segundo grupo fue denominado "fuera de serie" en los cuales, de acuerdo con las experiencias mencionadas por los usuarios, hay un constante abuso físico y psicológico; las condiciones al interior las reportan como precarias y es común que exista una falta de estructura en las actividades cotidianas.

En su mayoría, los entrevistados señalaron haber vivido múltiples internamientos, por lo que tuvieron la oportunidad de realizar una comparación en cuanto a la experiencia vivida en los llamados centros "light" como en los "fuera de serie", evidenciando en estos últimos el uso de violencia como medio de control y sometimiento ante la resistencia manifestada por el usuario.

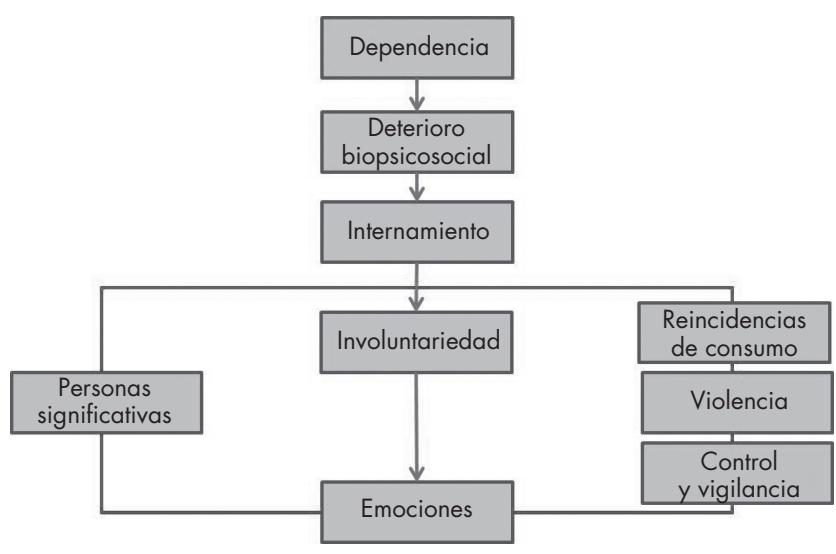

Figura 2. Proceso de Internamiento. 
"A mí se me maltrató mucho... me rompieron las costillas, me humillaban, me golpeaban, me bañaban con agua fría... me hicieron muchas cosas..." (José Ramón, 35 años).

"...viví muchas agresiones, me violaron, me metieron un palo de escoba por el ano, hicieron muchas cosas muy malas... recuerdo que bañaron a un cuate durante toda la noche, lo dejaron en la regadera con agua helada, luego lo levantaron a las siete de la mañana con unos ventiladores, y para las dos de la tarde se estaba muriendo de pulmonía... falleció y no les importó." (Roberto, 53 años).

En comparación, los participantes refirieron que los centros "light" se caracterizan por la implementación de una interpretación tergiversada de la filosofía de los 12 pasos de AA. No obstante buscan en apariencia el respeto a la integridad y dignidad del usuario aunque emplean estrategias de confrontación, aparentemente reguladas, que están dirigidas a romper las defensas psicológicas, la ingobernabilidad y soberbia de los usuarios, intentando con esto implantar un pensamiento 'humilde, moldeable y espiritual'.

"En este centro nadie me pega, no me tienen 12 o 16 horas sentado en una silla escuchando juntas y juntas y juntas, que no me enseñan nada... aquí me han llevado a tener conciencia de mi enfermedad, he aprendido a conocerme a mí mismo, he encontrado la esencia de la espiritualidad, que consiste en servir a los demás, pues eso me hace sentirme útil... Además aquí se come bien, el trato es diferente, la gente piensa y actúa diferente. Los compañeros que van adelante dan el ejemplo y van demostrando que sí se puede; pues han enfrentado situaciones de vida tan adversas como yo y las han superado. Ellos cada día me ponen la pauta para seguir adelante y esa es la gran diferencia entre un anexo ("fuera de serie") y esto ("light")." (Ricardo, 53 años).

Por otro lado, se puede apreciar en los relatos que el concepto de "humildad" es imprescindible en todo el proceso, ya que éste es empleado por "servidores y padrinos" para tratar y adoctrinar al interno. La "humildad", de acuerdo con la concepción de los entrevistados, consiste en depositar la confianza en un "poder superior", mismo que les permitirá mejorar su "espiritualidad" y encontrar la fuerza para lograr la abstinencia y mantenerla a largo plazo.

En las entrevistas se encontró que los centros denominados "light", promueven en su mayoría la conciencia de las razones por las cuales el usuario desarrolla la dependencia al alcohol u otras drogas, por medio de un diálogo confrontativo y rudo, cuyo objetivo es moldear la congruencia entre "los juicios y las actitudes", estrategia que parece estar fundamentada en la inserción del sentimiento de culpa. Por otra parte, se reporta que los centros "fuera de serie", utilizan la violencia como el método de aprendizaje para el desarrollo de la "humildad", situación que parece estar fundamentada en la provocación del miedo más que en el desarrollo de una conciencia real. Bajo esta postura, se piensa que la soberbia conduce al usuario a creer que puede tener el control sobre las sustancias y, por tanto, la nulidad del individuo le permitirá desarrollar la humildad y finalmente alcanzar la recuperación.

"Ah, bueno desde que llegué me recibieron con muchas groserías, me golpeaban, me sometían a estar en muchas juntas todos los días durante todo el día... En las noches me llegaron a dejar de pie, sin dormir y sin comer.
Después del tercer día ya empezaba a delirar... y terminaron por ponerme a servir café vestido de mujer y así me enseñaron, según ellos, a ser humilde." (Ricardo, 23 años).

Otro aspecto de relevancia para evaluar la experiencia en los CRAMAA "fuera de serie" son las estrategias por medio de las cuales se mantiene el orden y la organización, así como las consecuencias ante la violación de los lineamientos de conducta establecidos. Los castigos, también llamados "aplicaciones", se hicieron evidentes durante el desarrollo de las entrevistas, pues, a decir de los entrevistados, las "aplicaciones" surgían como resultado de una conducta o actitud considerada inapropiada, aunque en la mayoría de los centros se hizo evidente la carencia de un reglamento que fungiera de referencia para definir e identificar la conducta no deseada.

\begin{abstract}
"después de un rato me pasaron a la sala donde hacen según las juntas, me sentaron en una silla de la cual no me podía mover y me obligaban a estar con las manos estiradas, y cuidadito y me quedara dormido pues manejan todo con golpes y me ponían una 'aplicación' como a hacer lagartijas con tabiques en la espalda mientras me daban de patadas." (Cristian, 24 años).

"pues entre golpes, cachetadas y patadas, a veces me ponían hincado en corcholatas, parado de cabeza o lo que su imaginación les dijera." (Joel, 24 años).

"Como tres veces me metieron en un tambo de agua fría, una vez cuando me sacaron, me metieron desnudo al baño y me dijeron: 'a ver... pon las manos en la pared' y pues obedecí; y me aventaban latigazos de agua fría... después, uno de ellos me ordenó que me secara y me dejó de pie toda la noche y cuando casi iba a amanecer me dieron un pants todo roto, una camisa que nada más tenía una manga y un suéter; entonces, por instinto me comencé a vestir, cuando llegó otro tipo y me dio una cachetada, diciéndome: '¿quién te dijo que te cambiaras?', a lo que contesté: 'pues ya me dieron la ropa...' y me respondió: 'te la dieron pero ¿quién te dijo que te cambiaras?' y me volvieron a castigar..." (Pablo, 39 años).
\end{abstract}

En el caso de los centros "light" no existen las "aplicaciones" pero los castigos se relacionaron con actividades de aseo o servicio en las instalaciones de los centros. Es evidente que no recurren al uso de violencia física, pero ante el incumplimiento de una regla, los "servidores y padrinos" utilizaban el diálogo confrontativo y rudo para fomentar en el otro la responsabilidad de sus actos.

La evaluación que realizan los entrevistados a su experiencia de internamiento depende en mucho del tipo de centro en el que hayan sido ingresados. Sin embargo, se observa un constante malestar provocado por la reclusión, percibida como una privación de la libertad, transgresión de su integridad y derechos básicos, lo cual los coloca en una postura de vulnerabilidad e indefensión.

"Realmente la experiencia de los 'fuera de serie' no es grata en ningún momento y a veces parecen historias de horror o parecen cuentos, pero son realidades... a mí me lastimaron muchísimo en los anexos, me hicieron mucho daño y yo no puedo entender cómo pueden existir lugares como esos." (Ricardo, 53 años).

Finalmente, los entrevistados señalaron que una de las funciones que cumple el internamiento es la contención, ya que éste les permite estar alejados de las personas, lugares y circunstancias relacionadas con el consumo, e incluso, de la disponibilidad de la sustancia misma. Además, el estar 
Cuadro 1. Experiencia en los CRAMMAA

\begin{tabular}{|c|c|}
\hline "Fuera de serie" & "Light" \\
\hline Se consideran 90 días de internamiento obligatiorio & Se consideran 90 días de internamiento voluntario \\
\hline $\begin{array}{l}\text { Se utiliza la violencia como método de aprendizaje pro- } \\
\text { visto por los "padrinos" para fomentar la "humildad" }\end{array}$ & $\begin{array}{l}\text { Para el fomento de la "humildad" se emplea el diálogo } \\
\text { confrontativo y rudo }\end{array}$ \\
\hline $\begin{array}{l}\text { Terapia "egorreductora" a través de un lenguaje imperati- } \\
\text { vo y altisonante, así como del sometimiento psicológico }\end{array}$ & $\begin{array}{l}\text { Terapia "egorreductora" a través de un lenguaje imperati- } \\
\text { vo y altisonante, así como del sometimiento psicológico }\end{array}$ \\
\hline Condiciones de violencia física, verbal y psicológica & Uso de lenguaje altisonante, pero no violencia física \\
\hline $\begin{array}{l}\text { Los "servidores y padrinos" utilizan las amenazas y fo- } \\
\text { mentan el miedo como una forma de control }\end{array}$ & No se hace uso de amenanzas ni amedrentamiento \\
\hline Estancia involuntaria & Estancia voluntaria \\
\hline $\begin{array}{l}\text { Castigos o "aplicaciones", predominantemente violentos y } \\
\text { que en ocasiones ponen en riesgo la integridad del usuario }\end{array}$ & $\begin{array}{l}\text { Castigos a través de actividades extra de aseo o servicio } \\
\text { al interior del centro }\end{array}$ \\
\hline $\begin{array}{l}\text { Confrontación directa, uso de castigos y abuso físico, psi- } \\
\text { cológico y sexual }\end{array}$ & $\begin{array}{l}\text { Confrontación regulada, de ideas y valores que mantie- } \\
\text { nen la conducta de consumo y no son compatibles con la } \\
\text { ideología promovida por el modelo de AA }\end{array}$ \\
\hline $\begin{array}{l}\text { Sometimiento y control justificado por la tergiversación } \\
\text { de la filosofía de los } 12 \text { pasos }\end{array}$ & $\begin{array}{l}\text { Interpretación de la filosofía de los } 12 \text { pasos, a través del } \\
\text { diálogo confrontativo, la persuación y la reflexión }\end{array}$ \\
\hline
\end{tabular}

recluidos facilita la desintoxicación, lo cual genera una mayor claridad en el pensamiento y permite que los usuarios evalúen la problemática de consumo y el deterioro en las diferentes áreas de vida. Sin embargo, también mencionaron que las ventajas del internamiento generalmente son percibidas mucho tiempo después (cuadro 1).

"El único beneficio era la contención, era imposible no drogarme ahí afuera; por eso, el beneficio que tuve fue mantener mi abstinencia treinta días, y pues como consecuencia estuve desintoxicado y pude tomar la decisión de quedarme aquí." (José Ramón, 51 años).

\section{D) Uso de servicios}

Un aspecto fundamental de la investigación fue conocer el funcionamiento de los CRAMAA y los servicios disponibles, así como la administración de los mismos. Por tanto, una parte de la entrevista fue dedicada a conocer dichos aspectos, enfatizando las áreas fundamentales para una recuperación, especialmente necesidades básicas de alimentación, descanso e higiene.

En términos generales, se reporta que la infraestructura que poseen los CRAMAA depende en gran medida de los recursos o apoyos que tengan, aunque en su mayoría se encuentran en condiciones de hacinamiento debido a la fuerte demanda de atención para las adicciones, el reducido número de instituciones en el sector público y los elevados costos de tratamiento en el sector privado.

Las instalaciones de los centros "fuera de serie" son descritas en general como una casa habitación donde conviven entre 80 y 120 personas, por lo que en ocasiones los usuarios deben dormir en el piso o incluso de pie. En ambos tipos de centros hay una sala para llevar a cabo las juntas de AA. Sin embargo, debido a lo reducido de las instalaciones, un espacio puede cumplir diferentes funciones según la necesidad del centro, por lo que éste puede convertirse en dormitorio, comedor e incluso baño.

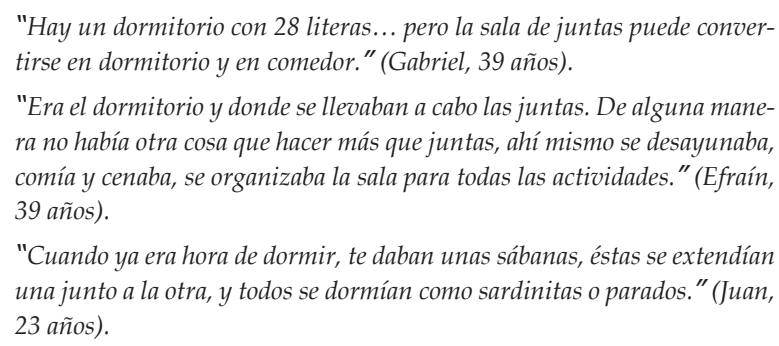

En el curso de las entrevistas fue frecuente encontrar la referencia a la pésima calidad de los alimentos. A decir de los entrevistados, la alimentación principal en los "fuera de serie" es el llamado "caldo de oso", hecho a base de cebolla y ajo con algunas verduras en estado de descomposición. Por otro lado, mencionaron que muchas veces las condiciones en las que se provee el alimento son deplorables, ya que además de no contar con un lugar destinado para ello, tampoco los utensilios que se les proporcionan cuentan con las condiciones mínimas de higiene.

"...el caldo de oso es agua hervida con cebollas y ajo... y una que otra verdurilla por ahí flotando, ¿no? Pero más bien son cebollas con, no quizá un pedacito de zanahoria y un pedacito de calabaza en tu plato, ¿no? Que no es un plato, es un traste en el cual tomas agua, comes y haces del baño..." (Tío, 45 años).

A diferencia de los "fuera de serie", se menciona que en los centros "light" la comida se encuentra en buenas condiciones además de ser suficiente para todos los usuarios y proveerse tres veces al día.

"Aquí (centro "light") tienes tus tres comidas, tu alimentación es de primera calidad, entre todos los internos mantenemos el servicio de la casa, unos se encargan de la limpieza, otros del abasto, otros de la cocina, mantenimiento... aquí no hay maltrato físico ni verbal... Es una estancia agradable..." (Gaby, 31 años). 
"Es un lugar limpio... se sirve una comida de muy buena calidad... se hacen las tres comidas todos los días y éstas son variadas y balanceadas, nos sentamos en una mesa como debe de ser..." (José Ramón, 51 años).

En cuanto al funcionamiento de los sanitarios, se mencionó que existe una diferencia importante entre los centros "light" y los "fuera de serie", ya que los primeros, en general, cuentan con las instalaciones necesarias, además de mantenerlos en regulares condiciones de higiene. Sin embargo, en el caso de los "fuera de serie", frecuentemente se hace referencia a la insuficiencia o ausencia de estos servicios; es por esto que destinan cubetas o incluso bandejas, donde el usuario debe realizar sus necesidades $y$, en ocasiones, hasta comer en la misma.

"¿Los baños?... Pésimos ¿cuál higiene puede haber cuando tienes un sanitario para 270 personas y tienes que orinar o defecar en una cubeta de 19 litros?" (Roberto, 53 años).

"...Tu plato... es un traste en el cual tomas agua, comes y haces del baño..." (Tío, 45 años).

El mantenimiento de la higiene se encuentra vinculado con la experiencia y el espacio de los baños. De acuerdo con lo reportado por los entrevistados, el aseo personal al interior de los CRAMAA muchas veces se veía limitado por la falta de recursos, además de que en ocasiones no se les proporcionaban los artículos de aseo personal, lo cual impactaba negativamente en su salud y calidad de vida al interior de los centros. Finalmente, durante las narraciones se hizo evidente la carencia de artículos personales de higiene, entre ellos, cepillos de dientes, jabón, toallas o rastrillos, además de que en ocasiones dichos objetos debían compartirse con todos los usuarios del centro (cuadro 2).

\footnotetext{
"Los 18 días que estuve en ese'anexo' fue sin lavarme los dientes ni bañarme porque, bueno, sí nos dejaban bañar diario pero no con agua caliente y por otro lado, me dieron ropa limpia después de 10 días que estuve ahí ..." (Gabriel, 39 años).

"Pasábamos de cinco en cinco a los baños y sólo funcionaban dos regaderas; pasábamos formados, nos mojábamos y nos íbamos pasando el jabón de uno a uno y ya después salíamos del baño." (Efrain, 39 años).

"...la higiene en el fuera de serie no era buena... muchas veces no había jabón para asearte y habían ¿cuántos rastrillos? Éramos qué... cerca de 70 compañeros y sólo teníamos tres rastrillos." (Pablo, 39 años).
}

\section{CONCLUSIONES}

En la actualidad se cuenta con cerca de 500 centros ambulatorios que integran la red de prevención y tratamiento del país (CIJ, CENADIC y otros). Sin embargo, es importante considerar que dichos centros no están preparados para atender trastornos graves de dependencia a sustancias, mismos que requieren atención hospitalaria, en función de las condiciones físicas y psiquiátricas del paciente. No obstante, es importante señalar que sí existen centros residenciales públicos y privados en México. Sin embargo, resultan insu-

Cuadro 2. Uso de servicios

\begin{tabular}{|c|c|}
\hline "Fuera de serie" & "Light" \\
\hline $\begin{array}{l}\text { Alimentación escasa y de baja calidad, llamada por los } \\
\text { usuarios "caldo de oso" }\end{array}$ & $\begin{array}{l}\text { Alimentación suficiente, tres veces al día y más balan- } \\
\text { ceada }\end{array}$ \\
\hline $\begin{array}{l}\text { Los usuarios carecen de artículos personales para higiene } \\
\text { (cepillo de dientes, toallas, rastrillos) y sólo hay agua fría } \\
\text { para bañarse }\end{array}$ & $\begin{array}{l}\text { Los usuarios tienen artículos para aseo personal y agua } \\
\text { caliente para el baño }\end{array}$ \\
\hline $\begin{array}{l}\text { No cuentan con baños en forma, en ocasiones se les en- } \\
\text { trega una cubeta para defecar, orinar } y \text {, en ocasiones, } \\
\text { comer }\end{array}$ & $\begin{array}{l}\text { Cuentan con baño de regulares a aceptables condiciones } \\
\text { de higiene }\end{array}$ \\
\hline $\begin{array}{l}\text { Por lo regular, se trata de una casa habitación en condi- } \\
\text { ciones de hacinamiento (entre } 80 \text { y } 120 \text { internos) }\end{array}$ & $\begin{array}{l}\text { Cuentan con espacios más amplios, aunque algunas ve- } \\
\text { ces están en condiciones de hacinamiento }\end{array}$ \\
\hline $\begin{array}{l}\text { Los usuarios duermen de pie o sentados, también hay } \\
\text { privación de sueño para asistir a las juntas AA }\end{array}$ & $\begin{array}{l}\text { Los usuarios tienen una cama asignada y un horario para } \\
\text { el descanso }\end{array}$ \\
\hline $\begin{array}{l}\text { No proveen servicios médicos, psicológicos y/o psiquiá- } \\
\text { tricos }\end{array}$ & $\begin{array}{l}\text { Algunos cuentan con la participación de un médico gene- } \\
\text { ral para la revisión de pacientes y sólo en algunas excep- } \\
\text { ciones se han logrado incorporar servicios profesionales } \\
\text { especializados }\end{array}$ \\
\hline $\begin{array}{l}\text { Sólo hay un espacio, el cual es utilizado para juntas AA, } \\
\text { comer y dormir }\end{array}$ & $\begin{array}{l}\text { Cuentan con diferentes espacios para cada actividad y } \\
\text { tienen una sala de observación }\end{array}$ \\
\hline Carecen de un reglamento escrito & Tienen un reglamento escrito \\
\hline $\begin{array}{l}\text { No existe claridad en las actividades a realizar ni en los } \\
\text { horarios }\end{array}$ & $\begin{array}{l}\text { Cuentan con un "rol de actividades" en el que los usua- } \\
\text { rios deben involucrarse }\end{array}$ \\
\hline No se tienen tareas bien definidas & $\begin{array}{l}\text { Los usuarios realizan servicios diferentes durante su estan- } \\
\text { cia (limpieza, cocina, jardín, entre otros) }\end{array}$ \\
\hline No están certificados & $\begin{array}{l}\text { Sólo algunos cuentan con certificación de la Secretaría de } \\
\text { Salud }\end{array}$ \\
\hline
\end{tabular}


ficientes o, en su defecto, muy costosos para el grueso de la población afectada.

Es así que ante dicho escenario, los CRAMAA continúan siendo una opción de primera mano ante la escasez de centros residenciales especializados para la atención de las adicciones que se ajusten a las necesidades de la población, a pesar de las limitaciones en infraestructura física y humana que éstos puedan presentar.

Dado el panorama que se ha vislumbrado a lo largo del estudio, resulta imprescindible la vigilancia y supervisión en el funcionamiento de estos centros, ya que no existe un registro publicado y oficial de la totalidad de estos lugares.

Por tanto, se hace evidente la necesidad de regulación y capacitación del personal al frente de dichas agrupaciones a fin de asegurar el respeto de los derechos humanos, así como el cumplimiento de los lineamientos marcados por la Norma Oficial Mexicana NOM-028-SSA2-2009 para la Prevención, Tratamiento y Control de las Adicciones y la integración del tratamiento, a fin de garantizar la mejora en la calidad de vida del usuario y su familia.

Finalmente, y a manera de propuesta, los investigadores creemos que, en función de las aportaciones tanto positivas como negativas para la rehabilitación del paciente con abuso de sustancias, se hace relevante comenzar con la desestigmatización de los llamados "anexos". Es por ello, que nuestra propuesta es llamarlos Centros Residenciales de Ayuda Mutua para la Atención de las Adicciones (CRAMAA), ya que con esto se esperaría no sólo buscar una reestructuración de fondo, sino también una resignificación de forma, en función de los significados que el colectivo tiene en referencia a estos centros. Asimismo, es importante hacer hincapié para los legos y el público en general, la diferencia entre un CRAMAA y el esfuerzo de los Grupos Tradicionales, ya que aunque los primeros siguen la filosofía de los 12 pasos como método terapéutico, distan mucho de ser lo que en esencia es Alcohólicos Anónimos (Grupos Tradicionales).

\section{Limitaciones}

Los resultados evidencian la relevancia y aportación de los mismos como coadyuvantes para la toma de decisiones de los servidores públicos en salud. No obstante, los resultados se circunscriben al análisis en población masculina, por lo que resulta relevante extenderlo para conocer el mismo fenómeno en mujeres, ya que el género es un factor significativo que impacta en la gravedad de la adicción y de los trastornos comórbidos, situación que se traduce directamente en un mayor deterioro de las mujeres en referencia a los hombres. Además, de acuerdo con la evidencia disponible, las mujeres reportan necesidades específicas para su atención como el cuidado de los niños y enfermedades físicas propias de las mujeres.

Finalmente resulta atractivo y complementario conocer más al respecto empleando a las familias como portavoces de la experiencia de este fenómeno, aspecto que terminaría de formar y cerrar el conocimiento de la vivencia de los usuarios de los CRAMAA.

\section{AGRADECIMIENTOS}

Este proyecto fue aprobado y financiado por la División de Investigación y el Departamento de Psicología de la Universidad Iberoamericana Ciudad de México.

Asimismo se extiende un agradecimiento especial a los profesores e investigadores tanto de la Universidad Iberoamericana Ciudad de México como del Instituto Nacional de Psiquiatría Ramón de la Fuente, que apoyaron y aportaron su conocimiento para hacer posible este proyecto, así como a los participantes que fueron entrevistados y que compartieron su experiencia de vida.

\section{REFERENCIAS}

1. Encuesta Nacional de Adicciones 2008. México: Consejo Nacional contra las Adicciones, Instituto Nacional de Psiquiatría Ramón de la Fuente, Instituto Nacional de Salud Pública y Fundación Gonzalo Rio Arronte; 2010.

2. Medina-Mora ME, Natera G, Borges G, Cravioto P et al. Del siglo XX al tercer milenio. Las adicciones y la salud pública: drogas, alcohol y sociedad. Salud Mental 2001;24:3-19.

3. Encuesta Nacional de Adicciones 2011. México: Consejo Nacional contra las Adicciones, Instituto Nacional de Psiquiatría Ramón de la Fuente, Instituto Nacional de Salud Pública y Fundación Gonzalo Rio Arronte; 2012.

4. Danielsson AK, Romelsjö A, Tengström A. Heavy episodic drinking in early adolescence: Gender-specific risk and protective factors. Subst Use Misuse 2011;46:633-643.

5. Medina-Mora ME. Las adicciones: su situación actual. En: Berruecos L, Díaz-Leal L (eds.). Curso básico sobre adicciones. México: CENCA; 1994.

6. McGrady BS, Miller WR. Research on Alcoholics Anonymous: opportunities and alternatives. New Brunswick, NJ: Rutgers University Press; 1993.

7. Fiorentine R. Alter drug treatment: Are 12-step programs effective in maintaining abstinence? Am J Drug Alcohol Abuse 1999;25:93-116.

8. Humphreys K. Professional interventions that facilitate 12-step selfhelp group involvement. Alcohol Health Res World 1999;23:93-98.

9. Pulido MA, Meyers M, Martínez A. Algunos datos acerca del funcionamiento de una muestra de grupos de autoayuda en México. Revista Mexicana Investigación Psicología 2009;1:85-97.

10. Lile B. Twelve step programs: An update. Addictive Disorders Their Treatment 2003;2:19-24.

11. Rosovsky H. Alcohólicos Anónimos en México: fragmentación y fortalezas. Desacatos 2009;29:13-30.

12. Central Mexicana de Servicios Generales de Alcohólicos Anónimos, A.C. (2012) Consultado el 13 de noviembre de 2012: http://www.aamexico.org.mx/

13. Alcohólicos Anónimos, Sección México (n.d.) Consultado el 13 de noviembre de 2012: http://www.aa.org.mx/

14. Alcohólicos Anónimos 24 horas Terapia Intensiva (n.d.) Consultado el 13 de noviembre de 2012: http://www.aa24horas.com.mx

15. Grupos 24 horas Alcohólicos Anónimos (2012) Consultado el 13 de noviembre de 2012: http://wwww.aa24horas.com

16. Jóvenes Alcohólicos Anónimos (n.d.) Consultado el 13 de noviembre de 2012: http://www.jovenesaa.org

17. Narcóticos Anónimos Región México (n.d.) Consultado el 13 de noviembre de 2012: http://www.namexico.org.mx 
18. Pérez GB. ¿Hacia dónde avanza el modelo asistencial al drogodependiente? Trastornos Adictivos 2001;3:205-213.

19. Kelly JF, Yeterian JD. Mutual-help groups for dually diagnosed individuals: Rationale, description, and review of the evidence. J Groups Addiction Recovery 2008;3:217-242.

20. Humphreys K, Wing S, McCarty D, Chappel J et al. Self-help organization for alcohol and drug problems: Toward evidence -based practice and policy. J Subst Abuse Treat 2004;26:151-158.

21. Norma Oficial Mexicana NOM-028-SSA2-1999 para la prevención, tratamiento y control de las adicciones. México: Diario Oficial de la Federación, 15 de septiembre de 2000.

22. Secretaría de Salud. Programa de Acción Específico 2007-2012. Prevención y tratamiento de las adicciones. México; 2008.

23. Marín-Navarrete R, Benjet C, Borges G, Nanni-Alvarado $R$ et al. Comorbilidad de los trastornos por consumo de sustancias con otros trastornos mentales en Centros Residenciales de Ayuda-Mutua para la Atención de las Adicciones. Salud Mental 2013 (en prensa).

24. Ortiz A, Soriano A, Galván J, Rodríguez E et al. Características de los usuarios de cocaína, su percepción y actitud hacia los servicios de tra- tamiento. Suplemento de la Revista de Salud Mental (suplemento) 1997;20:8-14.

25. Romero M, Gómez C, Ramiro M, Díaz A. Necesidades de atención a la salud mental de la mujer adicta. Salud Mental 1997;20(2):38-47.

26. Hernández A, Galván J. Estudio sobre los antecedentes y redes sociales de un grupo de usuarios de drogas antes del ingreso a tratamiento. Cartel presentado en la XXI Reunión de Investigación del Instituto Nacional de Psiquiatría Ramón de la Fuente Muñiz; México; 2009.

27. Merton RK, Fiske M, Kendall PL. The focused interview. Glencoee III; Free Press; 1956.

28. Monahan K, Egan EA, Van Horn L, Arthur M et al. Community-level effects of individual and peer risk and protective factors on adolescent substance use. J Community Psychology 2011;39:478-498.

29. Feinberg ME. Community epidemiology of risk and adolescent substance use: Practical questions for enhancing prevention. Am J Public Health 2012;102:457-468.

30. Consejo Nacional Contra las Adicciones. Observatorio mexicano en tabaco, alcohol y otras drogas 2003. México: Consejo Nacional Contra las Adicciones, SSA; 2003.

Artículo sin conflicto de intereses 